\title{
THE USE AND EFFECTIVENESS OF CAREGIVER INTERVENTIONS FOR ALZHEIMER'S PATIENTS
}

\author{
Leva K. Swim and Randa L. Shehab \\ School of Industrial Engineering \\ University of Oklahoma \\ Norman, OK 73019
}

\section{BACKGROUND}

$\mathrm{AD}$ is a progressive brain disorder that results in memory loss, unusual behavior, personality changes, and a decline in cognitive abilities. The disease progression varies significantly from person to person. Typically, AD patients live for 4 to 8 years after diagnosis; however, the disease can continue for up to 20 years (National Institute on Aging, 1997). Currently, AD is irreversible.

Untrained caregivers (e.g., spouses, relatives, and friends) form the backbone of the informal system of longterm care for $\mathrm{AD}$ patients. The majority $(70.2 \%)$ of the caregivers are female and are either wives $(61.5 \%)$ or daughters $(36.6 \%)$ of the person with $\mathrm{AD}$. The median age of caregivers is 63.6 years and the median education is 14 years (Beisecker, Chrisman and Wright, 1997).

The emotional burden on caregivers can be tremendous as they struggle to cope with the variety of $\mathrm{AD}$ symptoms. Although there is a large amount of information available on $A D$, it does not reach many of the caregivers that could benefit from it. The information is not readily available nor is it easy to use.

\section{METHODOLOGY}

The research objective was to identify caregiver intervention strategies that are used to increase the quality of life for both $\mathrm{AD}$ patients and their caregivers. A survey was developed to use in interviewing $\mathrm{AD}$ caregivers. The survey structure was guided by a set of intervention strategies developed in part from Taft's (1995) taxonomy of caregiving approaches, which incorporates seven domains: social, psychological, functional, behavioral, environmental, medical, and cognitive. The purpose of the survey was to determine the frequency of use and effectiveness of intervention strategies proposed by Taft (1995) and other strategies identified in the literature. Four $\mathrm{AD}$ caregivers were interviewed regarding intervention strategies and their effectiveness. The interviews were conducted by telephone and took approximately 1 hour each.

\section{RESULTS}

The results of the survey were summarized in a comprehensive list of the specific interventions used by the caregivers with usage and effectiveness ratings for each intervention approach. Strategies used by all caregivers included relating, empathetic caring, supportive touch, being responsive, and helping to remember. The individual interventions that were rated the most effective (with an average rating of $5.0,5=$ very effective) were relating (social), validation therapy (psychological), and providing natural areas (environmental). The lowest average effectiveness rating was for the confrontation intervention (1.33).

Each caregiver interviewed used at least one intervention strategy from each of the social, psychological, behavioral, and cognitive domains. Social and psychological interventions were the most commonly used. One caregiver reported no use of environmental strategies and two reported no use of medical strategies. In addition, social interventions were perceived as most effective. Environmental strategies, although not as commonly used, were also perceived as highly effective.

Most of the interventions are successful at mild or moderate stages of AD. Social interventions, such as relating, empathetic caring and supportive touch, are recommended for all stages of $\mathrm{AD}$. Interventions to promote continued cognitive functioning are recommended for mild stages of AD. More behavioral and environmental intervention strategies are recommended for moderate stages of $\mathrm{AD}$.

\section{CONCLUSIONS}

The prevalence of $\mathrm{AD}$, the variation in patient symptoms, and the lack of primary caregiver training all support the need for continuing research on $\mathrm{AD}$ intervention strategies. This study indicated that AD caregivers use interventions based on intuition and general "parenting skills," such as "going along with" the patient and avoiding confrontation. Interventions that caregivers thought would be beneficial (although not necessarily used) included environmental interventions typically used for accommodating older persons (e.g., walk-in showers) and the more creative cognitive interventions (e.g., memory tapes). Participation in an Alzheimer's support group was also noted as important. However, physician involvement in linking caregivers to information was minimal. Intervention strategies that are more proactive and effective for all stages of AD must be communicated to caregivers so that the quality of life can be enhanced for the patient and the caregiver.

\section{REFERENCES}

Beisecker, A.E., Chrisman, S.K., Wright, L.J. (1997). Perceptions of family caregivers of persons with Alzheimer's disease: Communication with physicians, American Journal of Alzheimer's Disease, March/April, 73-83.

Taft, L.B. (1995). Interventions in dementia care: Responding to the call for alternatives to restraints. The American Journal of Alzheimer's Disease, March/April, 30-38.

National Institute on Aging (1997). Progress report on Alzheimer's disease. http:/www.alzheimers.org/pr97.html. 